\title{
A 4D-Optical Measuring System for the Dynamic Acquisition of Anatomical Structures
}

\author{
Kathleen Denis ${ }^{1}$, Tom Huysmans ${ }^{1}$, Tom De Wilde ${ }^{1}$, Cristian Forausberger ${ }^{1}$, \\ Walter Rapp ${ }^{1}$, Bart Haex ${ }^{1}$, Jos Vander Sloten ${ }^{1}$, Remi Van Audekercke ${ }^{1}$, \\ Georges Van der Perre ${ }^{1}$, Kjell Roger Heitmann ${ }^{2}$, and Helmut Diers ${ }^{2}$ \\ 1 Division of Biomechanics and Engineering Design, K.U.Leuven, \\ Celestijnenlaan 200A, B-3001 Leuven, Belgium \\ ${ }^{2}$ Diers International $\mathrm{GmbH}$, \\ Dillenbergweg 4, 65388 Schlangenbad, Germany
}

\begin{abstract}
This paper presents a novel measuring system for the detection of moving skeletal structures. The system uses white light raster line triangulation in combination with biomechanical modeling techniques. White light raster line triangulation visualizes surfaces (e.g. the back surface) in an accurate and repeatable way, without detrimental effects, and without making contact to the human body. By making use of modeling techniques such as active contour models, active shape models and inverse kinematic models, biomechanically relevant results such as the position of the skeletal segments during motion are obtained.
\end{abstract}

\section{Introduction}

\subsection{Clinical Relevance}

The number of people suffering from musculoskeletal complaints, such as low back pain, is huge. It is by far the most important cause for work absenteeism in Europe, and as the population is increasingly sedentary and ageing, it is a growing problem. Orthopedic physicians and physiotherapists are required to analyze a variety of movements to diagnose pathological or abnormal changes. Therefore, an objective measurement tool to provide the medical examiner or the physiotherapist with clinically relevant data to support the diagnosis and therapy plan is required.

\subsection{Innovation of the Measuring System}

Standard diagnosis methods for musculoskeletal problems consist of expensive medical procedures (e.g. CT scans), involving potentially harmful ionizing radiation, or require expert operator interaction (e.g. 3D motion analysis based on marker tracing). Most of these methods are aimed at static purposes only and do therefore not involve dynamic measurements. Furthermore they show problems in specific cases (e.g. shift of markers on skin in marker tracing systems). So far, low cost measurement devices for preventive screening, diagnosis or treatment 
indication and control are not available. The objective was therefore to develop a contact-free measuring system for body surfaces, which is suited for dynamical applications in order to measure the human body in motion. By reconstructing internal anatomical structures, such as the spinal column, the system incorporates functional analysis capacities for medical, ergonomic, and revalidation applications. The reconstruction of internal anatomical structures is based on biomechanical modeling. In this paper, the reconstruction of the spine during stepping and the reconstruction of the shoulder during arm abduction are shown as examples of these techniques.

\section{Materials and Methods}

\subsection{White Light Raster Line Triangulation}

White light raster line triangulation (WLRT) enables the scanning of objects in $3 \mathrm{D}$ by projecting raster lines on its surface and by capturing these lines under a known and fixed angle with a camera [1] (Figure [1). Based on triangulation algorithms, spatial coordinates of all raster points are calculated, resulting in a dense point cloud of randomly distributed points describing the measured surface. These data points are transformed to a regular grid by using interpolation, which will simplify further calculations. In this way, the system captures and analyzes body motion with a frequency of $15 \mathrm{~Hz}$.
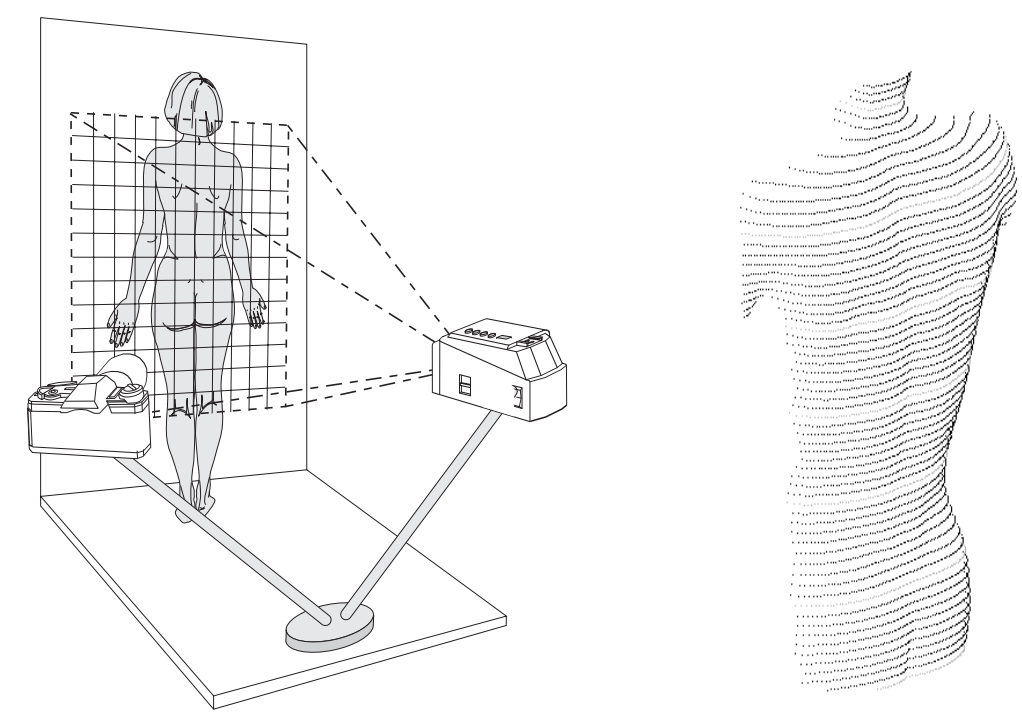

Fig. 1. WLRT acquisition (left) and point cloud describing the back surface (right). 


\subsection{Active Contour Models (ACM)}

The technique of active contours (ACM) or "snakes" was first proposed for outlining purposes [2]: a specific (mathematical) cost is defined in such a way that a minimization of the cost leads to an optimal recognition of a well defined feature. Starting from an initial estimation, the objective is to iteratively move the contour on the image until the cost is minimized and appropriate contour properties are achieved. The cost of an ACM comprises two parts: an external and an internal cost. The external cost guides the contour to a minimal cost position on the surface; the internal costs serve as a smoothness constraint and are defined in such a way that biomechanically incorrect solutions are excluded. The technique of ACM is applied to the extraction of information from the back surface. The external cost that is used for the detection of underlying skeletal structures in movement consists of a surface curvature cost and a symmetry cost. The internal costs include bending and torsion costs, an equidistance cost and a constraint cost. The optimization problem locates an outline with a minimal total cost, starting from an initial ACM.

\subsection{Active Shape Models (ASM)}

An active shape model (ASM) can be seen as "smart snake" that is only able to deform in ways characteristic of the class of objects it represents [3]. An ASM is built by learning patterns of variability from a training set of correctly labelled points on images. By examining the statistics of the positions of the labelled points a point distribution model can be derived. The model gives the average positions of the points, and has a number of parameters which control the main modes of variation found in the training set. In this way the developed model can only deform in ways found in the training set.

\subsection{Inverse Kinematic Models (IKM)}

For the shoulder joint, a lot of the skeletal information is hidden underneath the back surface. To reveal this information, a model has been developed to be used together with the surface measurements. The skeletal model contains the sternum, clavicula, scapula and humerus. Between the bones, three joints have been defined: the sternoclavicular joint, the acromioclavicular joint and the glenohumeral joint. These joints are modeled as three-degrees-of-freedom spherical joints. The scapula moves freely with respect to the thoracic wall, to permit scapular winging, which is a condition sometimes caused by nerve injury or another disorder affecting the shoulder. The model has been parameterized; it contains one parameter per bone. The motion of the links is limited in the spherical joints, using limits obtained from biomechanical literature 44. The bones have been modeled as geometrical primitives. Surface geometry has been added to the model for visualization purposes. Figure 2 shows both. 

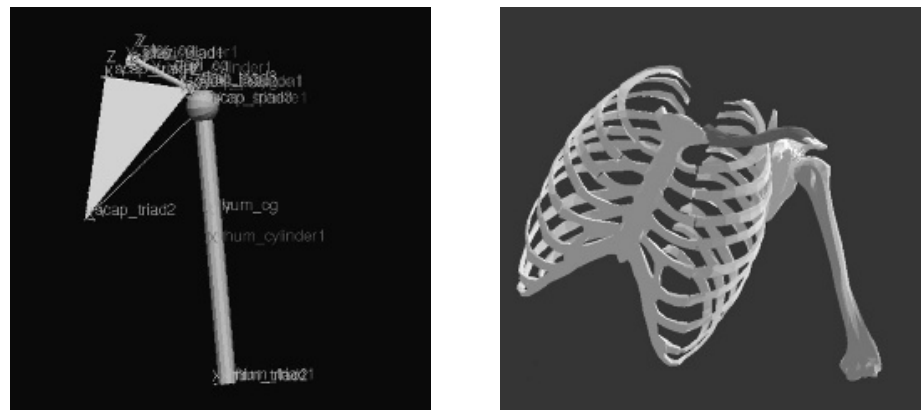

Fig. 2. Shoulder model consisting of geometrical primitives (left) and with surface geometry (right).

\section{Results}

\subsection{Example 1: ASM for the Detection of the Pelvis for a Stepping Motion}

ASM's were derived for an objective localization of anatomical landmarks which correspond to the vertebra prominens, the posterior superior iliac spines on the pelvic bone (os ilium) and the sacrum point (the beginning of the rimi ani). At first a point distribution model (PDM) is derived for stepping, based on a few training sets of the movement. The PDM showed clear relations between the anatomical landmarks. Figure 3 shows the first mode shape for a stepping movement. This single mode shape already explains $90 \%$ of the total variation in $3 \mathrm{D}$. The PDM is then used to locate the anatomical landmarks on persons who performed a similar movement (ASM). The detection of the four anatomical landmarks for three time steps, using this ASM, is indicated on figure 4



Fig. 3. First mode shape for stepping 

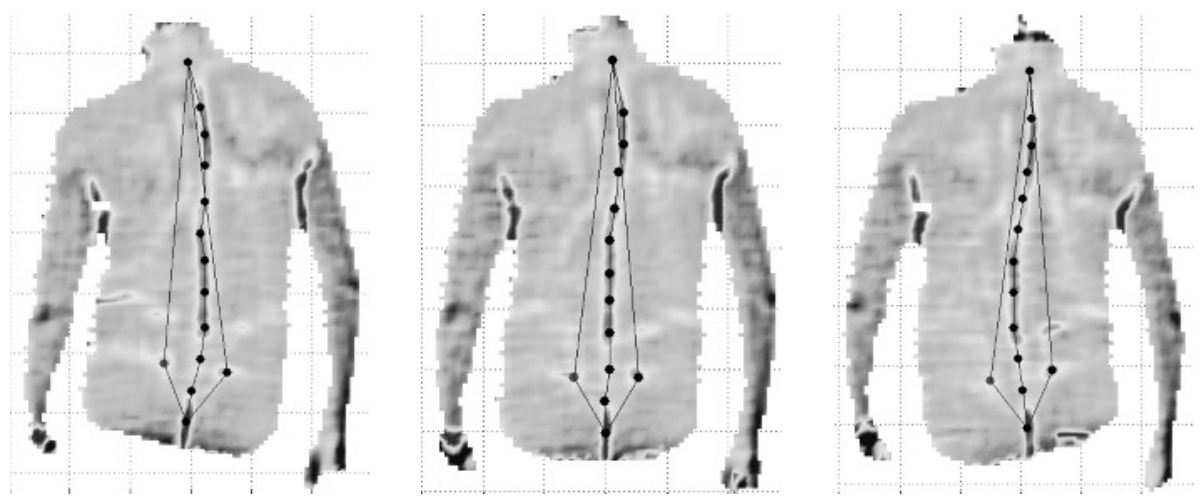

Fig. 4. Detection of the pelvis and the line through the spinous processes based on an $\mathrm{ACM}$ and an ASM (stepping, three time steps)

\subsection{Example 2: ACM for the Detection of the Line Through the Spinous Processes}

The detection of the line through the spinous processes over different measurements can only be made if the cost function is composed of invariant shape properties. Surface curvatures, for example, are an invariant property of the back shape, and can thus be used to analyze the shape of the human back. As a consequence, the positions of the detected anatomical landmarks are independent of the patient's position. An ACM is used for the detection of the line through the spinous processes. The ACM makes use of an asymmetry function, comprising the major part of the external cost for the active contour. The mean curvature is also included - as a minor factor - in order to improve the overall estimation of the line, e.g. to overcome local problems. In order to avoid results that are impossible from a biomechanical point of view, internal costs are added. At first bending and torsion costs are included; these terms are related to the smoothness of the curve, preventing the curve from physically impossible positions. Furthermore, active contour points will mount up during the calculations at places with a high surface curvature and/or symmetry. To avoid this effect, a final internal cost is included to keep all active contour points at an equal distance. A spline describes the ACM. Based on this spline, the internal spine is reconstructed using an anatomical formula, estimating the distance of the skin to the center of a vertebral body [1]. Figure 4 shows the detected line through the spinous processes with the ACM's for different time steps (stepping motion).

\subsection{Example 3: IKM for the Detection of the Scapula During Arm Abduction}

In case the measurements do not show enough information, an IKM is used. The shoulder IKM is used as follows: in preliminary measurements the person performs standard motions from which the parameters are quantified. After this, 

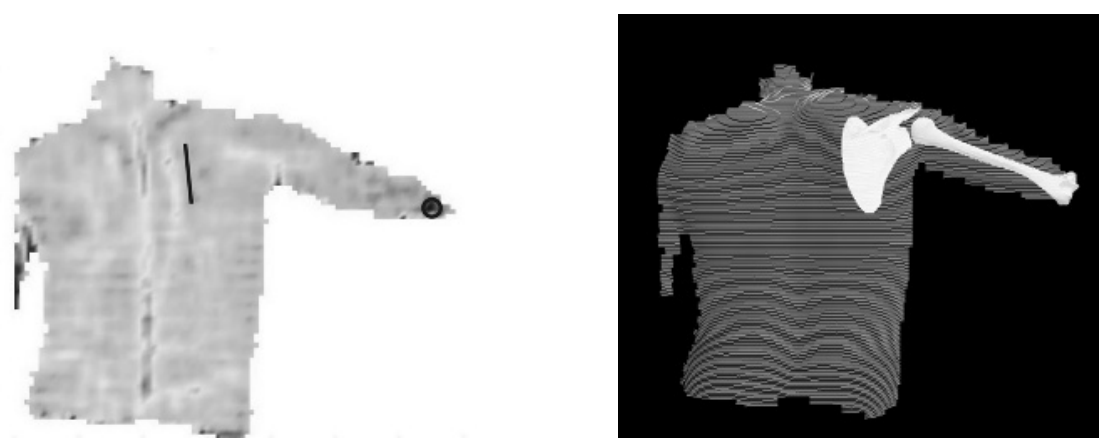

Fig. 5. The mean curvatures of one image from a dynamic sequence, together with the reconstruction of the humerus and scapula.

during the actual measurements, the model is used as extra information. The model calculates the shoulder position using inverse position kinematics. In a dynamic sequence, the model predicts the next position. When an image does not contain the information needed to detect the positions, the information is updated with the model prediction. Figure 5 shows the reconstruction of the scapula and the humerus in one image of a dynamic sequence.

\section{Presentation of the Results}

Figure 6 shows the user interface to view the results of a dynamic measurement. The left window is the 4D-(three dimensional and time dependent)surface window, the right window gives a graphic representation of selected parameters as a function of time. In the left window, succeeding images of the back surface are shown, with the possibility to show the raster lines, point cloud, mean curvatures, Gaussian curvatures, and 3D-inner skeletal structures in different views. The right window shows clinical parameters such as (for the trunk) trunk imbalance, trunk torsion, trunk inclination, (for the pelvis) pelvic tilt, pelvic torsion, (for the spine) lordotic angle, kyphotic angle, (for the scapula) maximum scapular winging, etc. In this example, the left window shows the Gaussian curvatures of a back surface in frontal view and the lines connecting the left and right dimples with the vertebra prominens and the sacrum point. The right window shows the pelvic tilt and the trunk imbalance as a function of time.

\section{Discussion}

Systems to detect skeletal structures in movement are mostly marker-based. The described 4D optical measuring system has the following advantages over these systems: there is no preparation time to equip the patient with the markers and there is no expert needed to palpate the patients and find the optimal positions of the markers. No contact is made with the patient, which is more agreeable for 


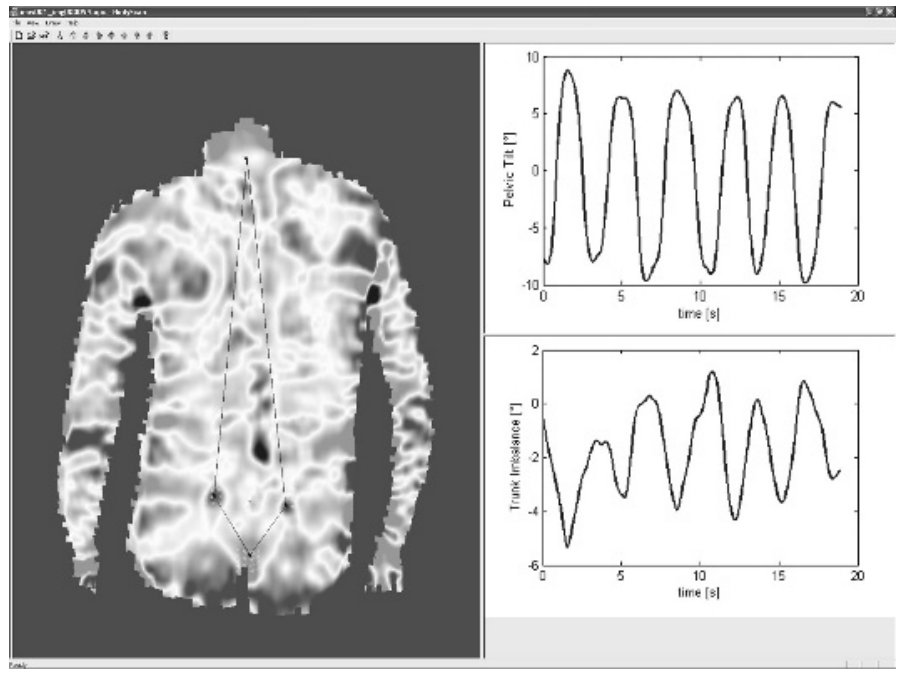

Fig. 6. A screen plot of the presentation of the results.

the patient. This system offers extra information, such as axial rotation of the vertebrae from back shape data. The marker-based systems have the disadvantage that during certain motions the skin with the markers shifts over internal skeletal structures, e.g. the shift of the skin over the scapula during arm elevation, resulting in a non-unique relation between marker and bone position. This source of errors does not exist in the present system. Compared to a markerbased system, the presented system has a limited field of view due to the raster projection, hence limiting the motions that can be investigated. For the moment, a field of view with a width of $1.5 \mathrm{~m}$ and a depth of $0.5 \mathrm{~m}$ is available. With the current frequency of $15 \mathrm{~Hz}$ it is not possible to investigate very fast motions yet; however, slow to medium-fast motions are not a problem. Increasing the frequency would mean an increase of data to be stored and of analyzing time. At present, both measurement time and analyzing time are short since automatic image analysis and processing are applied.

By making use of biomechanical models such as active contour models, active shape models and inverse kinematic models, biomechanically relevant results are obtained. The open structure of the ACM allows the addition of other biomechanical constraint costs, e.g. to examine specific etiological phenomena. In case of scoliosis research, for example, an extra cost is added, describing the observed relation between the lateral deviation and the axial rotation of the vertebrae.

The uniqueness of the presented system makes it difficult to compare the results in $4 \mathrm{D}$. However, the accuracy of the position of the internal skeletal structure has been checked for static purposes using radiographic scans. Drerup and Hierholzer developed an automatic detection of landmarks and spine for static purposes. They obtained an accuracy of $1.3 \pm 1 \mathrm{~mm}$ for the dimples and the vertebra prominens [5]. The position of the centre of the vertebrae is reconstructed with 
an accuracy of $3.9 \mathrm{~mm}$ [6], in comparison with radiographic scans. Compared with the method of Drerup and Hierholzer, the described system has a similar accuracy: a lateral deviation of the spine of $0.9 \mathrm{~mm}$ and an axial rotation of the spine of $0.4^{\circ}$ has been obtained [7], and the landmarks are positioned within $2 \mathrm{~mm}$. The main causes for the errors are the noise of the video images and the algorithmic estimation of the line through the spinous processes and of the vertebral rotation $\left( \pm 3^{\circ}\right)$; nevertheless the error is acceptable for the intended applications. The accuracy is thus better than with marker-based systems, with which the landmarks can be reconstructed with an accuracy of $4.2 \pm 1.7 \mathrm{~mm}$ [8].

\section{Conclusion}

The main advantages are the system's ability to reconstruct the internal skeletal structure, without the use of markers or potentially harmful or expensive equipment, as well as its ability to indicate and quantify pathological changes, both at an early stage and in connection with diagnosis and therapy. The contact-free 4D measurement, combined with the automatic detection of anatomical landmarks and the reconstruction of skeletal structures, makes the system unique. The measuring system can be used in public health centers, e.g. for the monitoring of scoliosis when bending sidewards. Ergonomic applications of the measurement device are envisaged as well, e.g. for work task analysis. Revalidation after sports injuries is another application area with a large potential.

Acknowledgement. This research has been supported by the cooperative research project under CRAFT (CRAF-1999-71293).

\section{References}

1. B. Drerup and E. Hierholzer. Back shape measurement using video rasterstereography and 3D reconstruction of spinal shape. Clin Biomech, 9:28-36, 1994.

2. M. Kass et al. Snakes: Active contour models. International Journal of Computer Vision, 1:321-331, 1988.

3. T.F. Cootes et al. Active shape models - their training and application. Comp Vision Image Underst, 61(1):38-59, 1995.

4. Engin and Tümer. 3D kinematic modelling of the human shoulder complex - part I. J Biomech Eng, 111:107-112, 1989.

5. B. Drerup and E. Hierholzer. Automatic localization of anatomical landmarks on the back surface and construction of a body-fixed coordinate system. J Biomech, 20:961-970, 1987.

6. B. Drerup and E. Hierholzer. Assessment of scoliotic deformity from back shape asymmetry using an improved mathematical model. Clin Biomech, 7:376-383, 1996.

7. T. Huysmans et al. 3D mathematical reconstruction of the spinal shape, based on active contours. J Biomech, accepted for publication, 2004.

8. N. Tardif et al. Evaluation of an integrated laser imaging/x-ray technique for torso asymmetry measurement in scoliosis. Arch Physiol Biochem, 108:200, 2000. 